doi:10.1016/j.vaccine.2006.02.026

Copyright @ 2006 Elsevier Ltd All rights reserved.

\title{
Differences of circulating Bordetella pertussis population in Argentina from the strain used in vaccine production
}

\author{
M. Fingermann ${ }^{a}$, J. Fernández ${ }^{a}$, F. Sisti ${ }^{a}$, M.E. Rodríguez ${ }^{b}$, B. Gattic, D. \\ Bottero $^{a}$ A. Graieb ${ }^{a}$, M.E. Gaillarda, S. González Ayalac, F.R. Mooi ${ }^{d,}$, H. \\ Lopardo $^{f, g}$ and D. Hozbor ${ }^{a, *}$
}

${ }^{a}$ Instituto de Bioquímica y Biología Molecular, Departamento de Ciencias Biológicas, Facultad de Ciencias Exactas, Universidad Nacional de La Plata, Calles 47 y 115 (1900) La Plata, Argentina

${ }^{b}$ Centro de Investigación y Desarrollo en Fermentaciones Industriales, CONICET, Facultad de Ciencias Exactas, Universidad Nacional de La Plata, Calles 47 y 115 (1900) La Plata, Argentina

'Laboratorio de Microbiología, Hospital de Niños Superiora Sor María Ludovica, Calle 14 No. 1631, La Plata, Argentina

'Laboratory for Vaccine-Preventable Diseases, National Public Health and the Environment, P.O. Box 1, 3720 BA Bilthoven, The Netherlands

eEijkman Winkler Institute, University Medical Centre Utrecht Heidelberglaan 100, 3584 CX, Utrecht, The Netherlands

fHospital de Pediatría Prof. Dr. Juan P. Garrahan, Pichincha 1850, Buenos Aires, Argentina

${ }^{9}$ Cátedra de Microbiología, Facultad de Ciencias Exactas, Universidad Nacional de La Plata, Calles 47 y 115 (1900) La Plata, Argentina

* Corresponding author. Tel.: +54 221425 0497x31.

\begin{abstract}
In Argentina, as in other countries, the number of pertussis cases has been increasing, even in highly vaccinated zones. Many reports suggest that the decline of vaccine efficacy due to antigenic shifts in the circulating Bordetella pertussis might be among the factors that contribute to pertussis re-emergence in different parts of the world. To evaluate the incidence of this factor in Argentina, we decided to characterize the circulating bacteria of an important demographic area of this country in comparison with the strain used for vaccine production. From 1997 to 2003 we collected nasopharyngeal samples from pediatric patients with signs of Bordetella infection hospitalized in the metropolitan area of Buenos Aires and La Plata, Argentina. From these samples we identified 28 B. pertussis, which were characterized by biochemical techniques, PCR, DNA fingerprint, prn and $p t x$ genes sequencing, and lipopolysaccharides (LPS) pattern. BOX-PCR from $B$. pertussis isolates yielded one cluster containing 13 isolates and some smaller ones, being all fingerprints different from the vaccine strain. Differences between Argentinean circulating bacteria and the vaccine strain were also observed for the Prn and Ptx variants as well as for the LPS pattern. Moreover, this last pattern seemed to change over the years. In addition, we identified two $B$.

bronchiseptica. The presence of this Bordetella species together with the observed differences between circulating $B$. pertussis and the strain used in vaccine production should be considered for the development of an improved vaccine.
\end{abstract}




\section{Introduction}

The genus Bordetella currently contains eight species, of which the three referred to as the classical Bordetellae, are closely related respiratory tract pathogens [1]. These three species, B. pertussis, B. parapertussis, and B. bronchiseptica, can be viewed as subspecies [2] and express similar virulence factors regulated by the BvgAS two-component system [3]. B. pertussis and B. parapertussis are human pathogens that cause whooping cough or pertussis in humans, a respiratory disease that is especially severe in young children. B. parapertussis has also been isolated from sheep but available data suggest that human and sheep strains are distinct and that transmission from sheep to humans does not occur [4]. $B$. bronchiseptica is an animal pathogen [5] but can also infect humans [6], [7] and [8].

From these three pathogens, $B$. pertussis causes the most severe disease in humans and because of that, attempts to develop vaccines for both treatment and prevention were made soon after $B$. pertussis was first isolated. The initial vaccines consisted of killed whole $B$. pertussis bacteria with which routine immunizations of children were started in the mid-1940s. In some countries this continues up to now and in some others acellular vaccines replaced whole cell vaccines. Although the introduction of both types of vaccines significantly reduced the incidence of pertussis, the disease is still endemic worldwide. Moreover, in some countries with highly vaccinated populations like The Netherlands [9], the United States [10], Canada [11], Sweden [12], and Australia [13] a reemergence of pertussis was recently reported. Several explanations have been suggested for the re-emergence of pertussis including changes in vaccine potency, decreased vaccine coverage, waning immunity and adaptation of the $B$. pertussis strains to vaccination. The latter hypothesis is mainly based on the finding of antigenic divergence between clinical isolates and the strains used in vaccine production. Genetic polymorphisms were mainly described for two of the main $B$. pertussis protective antigens, namely, pertussis toxin (Ptx) and pertactin (Prn) [14], [15], [16], [17], [18] and [19]. In particular, four Ptx S1 subunit variants (S1A-D) and 10 Prn variants (Prn1-9 and Prn11) were identified in the bacterial circulating populations [16] and [19]. These polymorphisms have been attributed to the ability of the bacterium to resist induced immunity, and cause an increase in the disease incidence [14] and [15].

In Argentina, the Public Health Ministry also registered an increase in pertussis incidence, even before improvements on diagnostic methodology were introduced. In this country whole cell pertussis vaccine was introduced 50 years ago and is still the only kind of pertussis vaccine being used. Up to now there are no data about the local circulating Bordetella strains in comparison with the strain used in vaccine production. Hence, it is of epidemiological interest to see whether the allelic frequencies in Argentinean Bordetella circulating strains are similar or not to the reported in the northern hemisphere. Therefore, we focused the present study on molecular characterization of Bordetella isolates obtained in a region of Argentina during the past 6 years. To our knowledge this is the first epidemiological molecular study on Bordetella performed in Latin America. 


\section{Methods}

\subsection{Patients, samples and bacterial growth conditions}

During the period 1997-2003 we collected nasopharyngeal samples from 400 hospitalized patients from Buenos Aires with signs of Bordetella infection. From these samples we searched for the presence of Bordetella spp. by specific PCR and culture. Bordetella was isolated on Bordet Gengou agar (Difco) supplemented with $15 \%(\mathrm{v} / \mathrm{v})$ defibrinated fresh sheep blood (BGA medium) at $36{ }^{\circ} \mathrm{C}$ for $72 \mathrm{~h}$.

To identify the Bordetella species causing the respiratory infection we performed discriminative PCR assays [20] and [21], biochemical typification using API 20 NE system (bioMerieux, Marci I'Etoile, France), and agglutination test with antiserum for B. pertussis and B. parapertussis (Murex Diagnostic, Dartfort England) on the clinical Bordetella spp. isolates.

All isolates were stored at $-80{ }^{\circ} \mathrm{C}$ in $0.9 \%(\mathrm{w} / \mathrm{v}) \mathrm{NaCl}$ containing $20 \%(\mathrm{v} / \mathrm{v})$ glycerol.

We also grew B. pertussis strain Tohama, and $B$. bronchiseptica strain CIP 9.73 (Collection de I'Institut Pasteur [CIP] designation) on BGA medium at $36^{\circ} \mathrm{C}$ for 48-72 h. In the experiments described below we used the Pasteur-MerieuxConnaught vaccine strain as a control since it is the vaccine strain used in Argentina.

\subsection{PCR for detection of B. pertussis, B. parapertussis and $B$. bronchiseptica}

Using the Fla1/Fla2 primers designed by us [20] we could detect the three species of Bordetella: B. pertussis, B. parapertussis, and B. bronchiseptica, and distinguish $B$. pertussis (size of amplified product $195 \mathrm{bp}$ ) from the other two pathogens (size of the amplicon $164 \mathrm{bp}$ ). As the sensitivity of this PCR is low for $B$. pertussis we also employed the oligonucleotide primers derived from the $p t x$ promoter (PT1/PT2) (size of the amplicon, $191 \mathrm{bp}$ ) designed by Grimprel et al. [21]. To discriminate $B$. bronchiseptica from $B$. parapertussis we used the primers Fla2/Fla3 [20]. All the oligonucleotides were provided by DNAgency Inc. (Malvern, USA). The PCR assays were performed as described earlier [19]. Positive PCR products were detected by ethidium bromide staining of $2 \%$ agarose gels after electrophoresis.

\subsection{DNA fingerprinting}

We performed total DNA amplification fingerprints using different primers BOXC1, BOXAR1, ERIC, MBOREP1, REP1 and NGREP2, whose sequences were reported by Versalovic et al. [22].

The deoxyoligonucleotide primers were synthesized by DNAgency (Malvern, PA, USA). We carried out PCR reactions in $25 \mu$ l containing: $50 \mathrm{mM}$ Tris, $\mathrm{pH}$ 8.3; $500 \mu \mathrm{g} / \mathrm{ml} \mathrm{BSA} ; 3 \mathrm{mM} \mathrm{MgCl} 2 ; 200 \mu \mathrm{M}$ dNTPs; $1 \mathrm{U}$ Taq polymerase (Promega Corp.); $10 \mu \mathrm{M}$ primer, and $2 \mu$ l of template DNA, previously obtained by phenol extraction. The amplifications were carried out in capillary tubes in an Idaho 1605 Air Thermo Cycler (ATC, Idaho Technology). The cycling conditions were as follows: $94^{\circ} \mathrm{C}$ for $2 \mathrm{~min}, 35 \mathrm{cycles}$ at $94^{\circ} \mathrm{C}$ for $10 \mathrm{~s}$, at $52{ }^{\circ} \mathrm{C}$ for $60 \mathrm{~s}$, and at $65^{\circ} \mathrm{C}$ for $8 \mathrm{~min}$ with a final cycle of $65^{\circ} \mathrm{C}$ for $16 \mathrm{~min}$. After the reaction, $10 \mu \mathrm{l}$ of 
the PCR products were separated in $1.5 \%$ agarose gels containing $0.5-1 \mu \mathrm{g} / \mathrm{ml}$ ethidium bromide, and photographed by using a digital camera Kodak DC290.

Computer-assisted analysis of the BOXAR1 fingerprint patterns was carried out using GelCompar software (Windows NT version 4.1, Applied Maths, Kortrijk, Belgium).

\subsection{Sequence analysis of the genes for pertactin and pertussis toxin $\mathbf{S 1}$ subunit}

We performed DNA sequencing of relevant regions of the prn and ptxS1 genes on $P C R$ fragments as described [16]. We used the primer pair AF/AR (5'-

CAATGTCACGGTCCAA-3'/5'-GCAAGGTGATCGACAGGG-3') for sequencing of prn region 1 , and for sequencing of prn region 2 the primer pair $B F / B R\left(5^{\prime}-\right.$ AGCTGGGCGGTTCAAGGT-3')/5'-CCGGATTCAGGCGCAACTC- $3^{\prime}$ ). For pertussis toxin, we used the primers S1-F2 ( $5^{\prime}$-CCCCTGCCATGGTGTGATC- $\left.3^{\prime}\right)$ and S1-R2 (5'-AGAGCGTCTTGCGGTCGATC-3') to amplify a 930 bp product, which contains the complete ptxS1 gene. We also used the S1-F2/S1-R2 for sequencing [18]. We confirmed mutations by sequencing using two additional primers: S1-MF/S1-MR (5'-ACAATGCCGGCCGTATCCTC-3'/5'-TTCGAAGTACGAGCTGGCGG-3').

\subsection{Immunoblots}

Cells corresponding to $2 \times 10^{10}$ colony-forming units of the Bordetella isolates were treated with Laemmli sample buffer and the extracts run on $12.5 \%(\mathrm{w} / \mathrm{v})$ polyacrylamide SDS-gels. After electrophoresis, we transferred the proteins from the polyacrylamide to a polivinylidenphosphate membrane (Immobilon P, Millipore) and incubated it with serum obtained from vaccinated patients, or with a 1:5000 dilution of mice polyclonal immune sera directed against AC-Hly (purified and subsequently inoculated into BALB/c mice as previously described [23]) or Ptx (kindly provided by Dr. Nicole Guiso, Laboratoire des Bordetelles, Institut Pasteur, Paris). In all the cases, we used alkaline-phosphatase-labeled sheep anti-mouse immunoglobulins to detect the presence of immune complexes.

\subsection{Lipopolysaccharide extraction and sodium dodecylsulfate- polyacrylamide gel electrophoresis (SDS-PAGE)}

Cells grown at $36^{\circ} \mathrm{C}$ in BGA medium were resuspended in PBS buffer, centrifuged $\left(10,000 \times g, 15 \mathrm{~min}, 4^{\circ} \mathrm{C}\right)$ and washed twice in distilled water. After adjustment of bacterial concentration, we extracted the lipopolysaccharides (LPS) by affinity chromatography as described [24]. We solubilized the isolated LPS by heating at $100{ }^{\circ} \mathrm{C}$ for $5 \mathrm{~min}$ in Laemmli sample buffer. We then applied the LPS suspensions to SDS-gels.

We performed electrophoresis at room temperature and constant voltage, and then visualized the LPS by the BioRad silver-staining technique.

\subsection{Peripheral blood polymorphonuclear leukocyte (PMN)}

We isolated peripheral blood PMN from heparinized venous blood of healthy human volunteers (age range: 25-45 years old) using Ficoll-Histopaque (SigmaAldrich, St. Louis, MO) gradient centrifugation. We harvested polymorphic leukocytes and removed the remaining erythrocytes by hypotonic lyses. Cell viability was $>99 \%$ as determined by trypan blue exclusion. Before functional assays, we washed twice the PMN with RPMI 1640 medium supplemented with 
$10 \%$ heat-inactivated fetal calf serum (FCS); the PMN were then resuspended, and used immediately. All experiments described in this study were conducted with freshly isolated PMN lacking FcaRI expression, as monitored by FACS analyses with FITC-conjugated anti-FcaRI mAb 22 [25].

\subsection{Phagocytosis}

We evaluated phagocytosis of $B$. pertussis and $B$. bronchiseptica as previously described [26]. To label bacteria for this study, we introduced into $B$. bronchiseptica CIP 9.73 and $B$. pertussis Tohama strains by conjugation the plasmid pGB5P1 that codes for $\mathrm{Km}$ resistance and for the green fluorescence protein. This plasmid was kindly provided by Dr. Allison Weiss (Department of Molecular Genetics, Biochemistry and Microbiology, University of Cincinnati). We grew GFP-expressing $B$. pertussis and GFP-expressing $B$. bronchiseptica overnight on BG agar plates and resuspended them in RPMI 1640 medium containing $10 \%$ FCS. Bacteria were incubated 30 min at $37^{\circ} \mathrm{C}$ with human sera $(2.5$ or $5 \%)$ obtained from a 6 years old child either before or 30 days after vaccination with whole cell pertussis vaccine After washing, we incubated the opsonized bacteria with phagocytic cells in a 70:1 ratio for $45 \mathrm{~min}$ at $4{ }^{\circ} \mathrm{C}$ to allow binding of bacteria to PMN. After extensive washing to remove nonattached bacteria, we divided the cells in two aliquots and further incubated them for $30 \mathrm{~min}$, either at $4{ }^{\circ} \mathrm{C}$ or $37^{\circ} \mathrm{C}$. Next, we detected remaining cell surface-bound opsonized bacteria by incubation ( 30 min at $4^{\circ} \mathrm{C}$ ) with Phycoerythrin (PE)-conjugated goat $\mathrm{F}\left(\mathrm{ab}^{\prime}\right)_{2}$ of anti-human IgG (from Southern Biotechnology Associates, Birmingham, AL). After washing, we analyzed the samples by flow cytometry. Five thousand cells were analyzed per sample. Green and red fluorescence intensities of cells maintained at $4^{\circ} \mathrm{C}$ throughout served as control for bacterial binding (i.e. $0 \%$ phagocytosis). The decrease in red fluorescence of green positive cells after incubation at $37^{\circ} \mathrm{C}$ reflects bacterial phagocytosis, as confirmed microscopically [26]. We calculated phagocytosis rates from the drop in mean red fluorescence intensity of greenpositive cells and expressed them in arbitrary units (AU), as described [27].

\section{Results}

\subsection{Description of samples and isolates}

For this study we collected nasopharyngeal samples over a period of 6 years from 400 hospitalized pediatric patients at the metropolitan area of Buenos Aires and La Plata (hereafter referred to as Buenos Aires). This important demographic region where an increase of pertussis cases was registered from 1999, concentrates $30 \%(11,500,000$ people $)$ of the total Argentinean population $(37,000,000$ people). The patients had signs of Bordetella infection with dry and emetic coughing for approximately 14 days. Apnea and cyanosis were frequent in infants younger than 3 months of age and sometimes necessitated intensive care in hospital. The age distribution of the patients was as follows: $90 \%$ younger than 1 -year-old, 7\% between 19 months and 3 years old, and only one patient 12year-old. The vaccination statuses of the majority of the patients were completed according to their age. Only for nine hosts the vaccination status was unknown. The vaccine used during 1997-2003 was the whole cell Pasteur-MerieuxConnaught vaccine and the vaccination schedule consisted in five doses given at $2,4,6$, and 18 months of age, and finally reinforcement at the age of 6 years.

From the collected samples, $25 \%$ were shown to be positive for specific Bordetella PCR [20] and [21] (98\% positive for B. pertussis and $2 \%$ for B. bronchiseptica). However, from the 100 PCR-positive nasopharyngeal samples, only 30 Bordetella isolates could be cultured on BGA plates. PCR assays using the flagellin promoter 
sequence as a target DNA region [20] showed that 28 out of the 30 isolates were $B$. pertussis (isolated from patients with ages ranging from 1 month to 3 years old) and the other two were $B$. bronchiseptica (isolated from vaccinated patients of 19 months and 12 years old). To confirm these PCR-based typifications, we analyzed oxidase, urease and motility by the API 20 NE system, and agglutination with antiserum for $B$. pertussis and $B$. parapertussis. The results obtained were coincident with those obtained with PCR assays.

\subsection{Virulence state}

We investigated the hemolytic activity of each clinical bacterial isolate in BGA and observed that all isolates were hemolytic and thus most likely virulent. Moreover, we evaluated the expression of virulence determinants by Western blot analysis using mouse polyclonal immune sera directed against AC-Hly and Ptx (data not shown). The $28 B$. pertussis isolates expressed both toxins and $B$. bronchiseptica isolates only expressed AC-Hly. The absence of Ptx expression by $B$.

bronchiseptica is consistent with previous studies [28].

\subsection{Lipopolysaccharide variation}

Phenotypic changes of surface bacterial components are commonly observed as a result of bacterial interaction with the host [29], [30], [31] and [32]. Among these surface components are the lipopolysaccharides, the major structural component of the outer membrane of Gram-negative bacteria and a highly immunogenic molecule. We here compared SDS-PAGE profiles of the LPS from clinical isolates and from the vaccine strain (Fig. 1). The LPS were extracted from cells grown in the same conditions. Vaccine strain LPS showed the typical twoband pattern, LPS A and LPS B. In turn, although the LPS from clinical isolates also showed a pattern with a preponderance of LPS A, some of them seemed not to have LPS B. This shift from the classical two-band pattern (LPS A and LPS B) to a single band (LPS A) occurred in the more recent isolates. In order to exclude possible artifacts due to growth and/or LPS extraction conditions we repeated the whole procedure three times for all samples starting from the glycerol stock, and the same results were consistently observed. We also analyzed the possibility that the absence of LPSB band could have been caused by a difference in LPS sample concentration loaded in the gel. However, similar LPS patterns in the SDS-PAGE were observed when four times higher concentration of LPS was loaded in the gel (not shown).

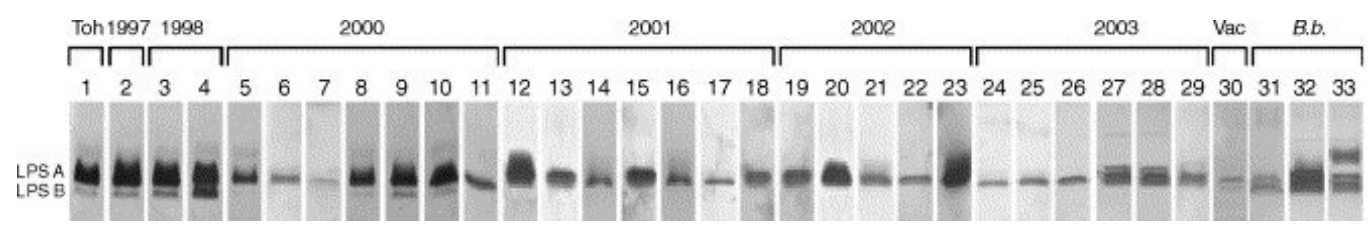

Fig. 1. Electrophoretic LPS profiles from different $B$. pertussis clinical isolates. LPS samples were separated in SDS-PAGE $(15 \%[\mathrm{w} / \mathrm{v}])$ and visualized via silver staining. The figure is a composite of several gels showing the LPS band patterns of each isolate. As internal standard Tohama and vaccine strain LPS were included in each gel. LPS samples in the gel correspond to the bacterial isolates obtained at the years indicated at the top of the figure. Tohama and B. bronchiseptica CIP 9.73 collection strains, and Pasteur-Merieux-Connaught vaccinal strain are also included in lanes 1, 31 and 30, respectively. This LPS analysis was repeated three times with the same result. 


\subsection{Sequencing of the structural genes encoding the S1 subunit of pertussis toxin and the repeated region of pertactin}

Polymorphism in the genes for pertactin ( $p r n)$ and the PtxS1 subunit ( $p t x S 1$ ) was studied in the 28 isolates of $B$. pertussis by DNA sequencing [16]. Our results on prn sequencing showed $B$. pertussis harboring prn2 variant to be prevalent in Buenos Aires (Table 1). In fact, of the 28 isolates studied $26(93 \%)$ contained the prn2 allele and $2(7 \%)$ the prn1/7 allele. Studies on PtxS1 subunit polymorphism showed that all $B$. pertussis isolated by us from 1997 to 2003 contained the ptxS1A allele. By contrast, the vaccine strain used in Argentina contains prn1 and ptxS1D alleles. Thus with respect to these two genes the vaccine strain was different than the prevalent circulating bacteria.

Table 1.

Frequencies of ptxS1 and prn alleles in different periods in Argentina

\begin{tabular}{|l|l|l|l|l|l|l|l|l|l|}
\hline Period & \multicolumn{1}{|l|}{ No. of B. pertussis clinical isolates with allele (\%) } \\
\hline & & \multicolumn{2}{l}{ ptxS1 } & \multicolumn{2}{l|}{ Prn } \\
\hline & & S1A & S1B & S1D & prn1 & prn2 & $\begin{array}{l}\text { prn3/ prn4/ } \\
\text { prn6 }\end{array}$ & prn7 & $\begin{array}{l}\text { prn8/ prn9/ } \\
\text { prn11 }\end{array}$ \\
\hline $\begin{array}{l}1997- \\
1998\end{array}$ & 3 & $3(100)$ & $\begin{array}{l}0 \\
(0)\end{array}$ & $\begin{array}{l}0 \\
(0)\end{array}$ & $0(0)$ & $3(100)$ & $0(0)$ & $0(0)$ & $0(0)$ \\
\hline $\begin{array}{l}2000- \\
2001\end{array}$ & 14 & $\begin{array}{l}14 \\
(100)\end{array}$ & $\begin{array}{l}0 \\
(0)\end{array}$ & $\begin{array}{l}0 \\
(0)\end{array}$ & $\begin{array}{l}2 \\
(14)\end{array}$ & $12(86)$ & $0(0)$ & $0(0)$ & $0(0)$ \\
\hline $\begin{array}{l}2002- \\
2003\end{array}$ & 11 & $\begin{array}{l}11 \\
(100)\end{array}$ & $\begin{array}{l}0 \\
(0)\end{array}$ & $\begin{array}{l}0 \\
(0)\end{array}$ & $0(0)$ & $\begin{array}{l}11 \\
(100)\end{array}$ & $0(0)$ & $0(0)$ & $0(0)$ \\
\hline
\end{tabular}

Tohama collection strain contained S1B and prn1; Pasteur-Merieux-Connaught vaccine strain contained $S 1 D$ and prn1.

\subsection{DNA fingerprinting}

The degree of genotypic similarity among the Bordetella clinical isolates was examined by a PCR-fingerprinting method. We tested the reproducibility of fingerprints with primers BOXC1, BOXAR1, ERIC, MBOREP1, REP1 and NGREP2 in PCR assays [22] using purified genomic DNA from four different isolates as templates in three independent experiments. With BOXAR1 primer we obtained the same results in each of the three independent reactions and in addition, the four fingerprint patterns could be easily distinguished from each other thus giving the best discriminatory results (data not shown). Based on these results the BOXAR1-PCR was selected for genotypic typing of the other clinical isolates.

Purified genomic DNA of the clinical isolates, the reference strains, or the vaccine strain, were used as templates in the PCR assays. PCR-amplified fragments were separated on an agarose gel (Fig. 2A) and the fingerprint patterns scanned into a computer. We used the GelCompar software package to derive phylogenetic relationships. The dendrogram derived is shown in Fig. 2B. BOXAR1 PCR of genomic DNA from all $B$. pertussis isolates yielded one major fingerprint profile and a small number of unique profiles. Clear differences in the band patterns between the vaccine strain and the isolates can be observed in the agarose gel. Small differences among the profiles of $B$. pertussis isolates can also be seen. 


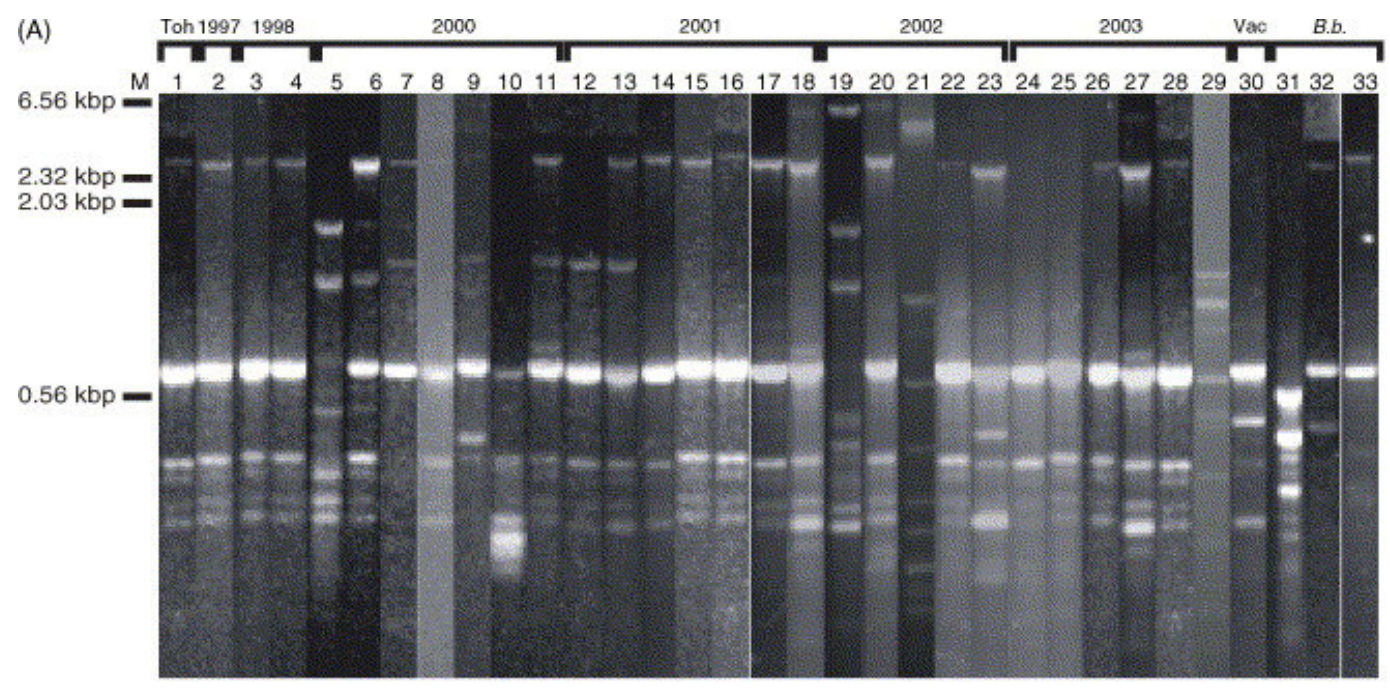

(B)
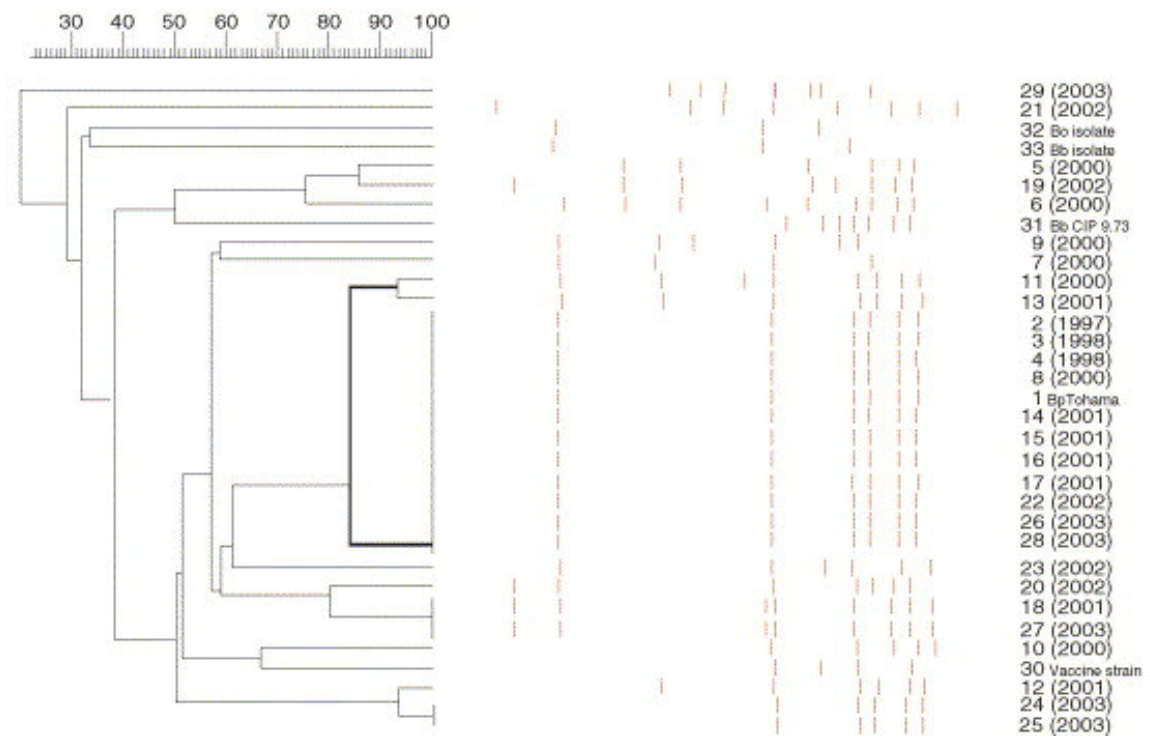

Fig. 2. Genotypic characterization of Bordetella clinical isolates. (A) BOXAR1-PCR fingerprint analysis of genomic DNA obtained from $B$. pertussis and $B$.

bronchiseptica clinical isolates. The figure is a composite of several gels showing the characteristic band patterns of each isolate. As internal standard Tohama and vaccine strain DNA were included in each gel. BOXA-PCR products derived from different templates were separated on a $1.5 \%$ agarose gel and stained with ethidium bromide. The year of isolation and the number of the isolate are indicated at the top of the figure. B. pertussis Tohama and B. bronchiseptica CIP 9.73 collection strains and Pasteur-Merieux-Connaught vaccinal strain are also included in lanes 1, 31 and 30, respectively. Lane labeled $M$ contain $\lambda$ HindIII size marker. (B) Dendrogram of $B$. pertussis clinical isolates BOXA-PCR fingerprint. The year of isolation and the number of the isolate are indicated at the right of the figure. The scale above the dendrogram describes percent relatedness of each branch of the dendrogram as determined by GelCompare software. This fingerprint analysis was repeated at least three times with the same result. 


\subsection{Cross immune protection between B. pertussis and B. bronchiseptica}

In order to investigate the cross-immune protection between $B$. pertussis and $B$. bronchiseptica we tested the reactivity of sera from a donor vaccinated with whole cell vaccine exhibiting high titers of anti-B. pertussis antibodies, as determined by ELISA, against whole cell proteins of both strains by western blot. As expected, this serum recognized polypeptides of both $B$. pertussis and $B$. bronchiseptica (not shown). However, the biological relevance of this cross reactivity has to be established. Opsonophagocytosis was found to be a key mechanism in preventing Bordetellae infection [26] and [27]. Determining whether anti-B. pertussis antibodies can act as opsonins for $B$. bronchiseptica might give a good indication of the biological activity of these antibodies in relation to $B$. bronchiseptica. Opsonophagocytosis of $B$. pertussis and $B$. bronchiseptica induced by serum obtained before (pre-immune) and after (postimmune) vaccination with pertussis whole cell vaccine was compared. Postimmune serum-induced efficient attachment and internalization of both Bordetella strains, as reflected by the high level of both the green and red mean fluorescence of PMN, as well as the drop of mean red fluorescence of PMN after incubation at $37^{\circ} \mathrm{C}$ (Fig. 3A). A positive correlation between serum concentration used for opsonization and bacterial phagocytosis was observed both for $B$. pertussis and $B$. bronchiseptica (Fig. 3B). Phagocytosis antibody dependency was further confirmed by the lack of opsonic activity by serum obtained prior vaccination (exhibiting no detectable antibody titers against $B$. pertussis as determined by ELISA). This was demonstrated by the low green and red fluorescence intensities of PMN incubated with either $B$. pertussis or $B$. bronchiseptica opsonized with pre-immune serum even at highest concentration (5\%) (Fig. 3). 


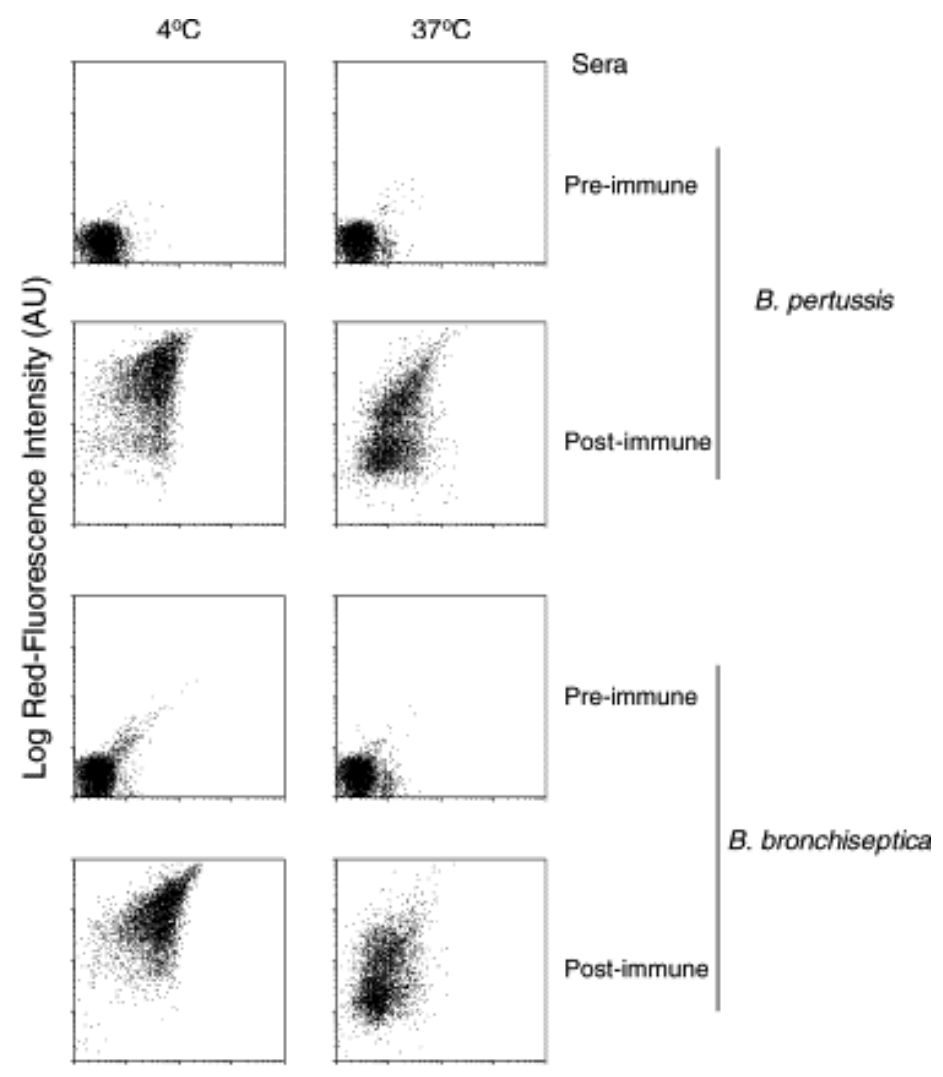

(A) Log Green-Fluorescence Intensity (AU)

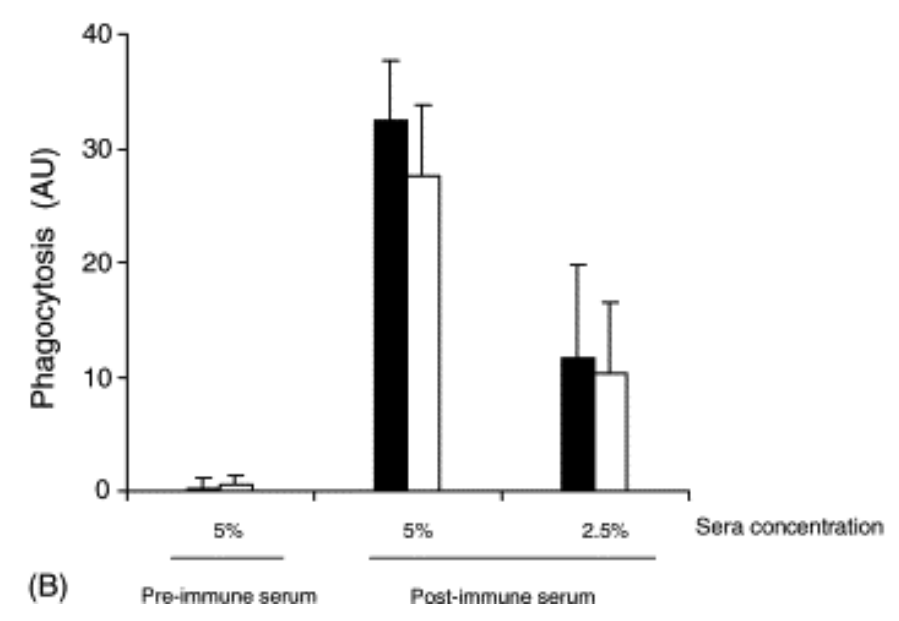

Fig. 3. Effect of antibodies induced by pertussis vaccination on $B$. pertussis and $B$. bronchiseptica phagocytosis by PMN. (A) PMN were incubated with either $B$. pertussis or $B$. bronchiseptica opsonized with serum ( $5 \%$ ) from a donor either before (pre-immune) or after (post-immnune) pertussis vaccination at $4{ }^{\circ} \mathrm{C}$ during $30 \mathrm{~min}$. Cells were split over two aliquots, and subsequently incubated for $30 \mathrm{~min}$ at either $4^{\circ} \mathrm{C}$ or $37^{\circ} \mathrm{C}$. Remaining surface-bound opsonized bacteria were detected by addition of PE-conjugated goat $F\left(a b^{\prime}\right)_{2}$ fragments of anti-human IgG antibodies. (B) PMN phagocytosis of $B$. pertussis (dark bars) and $B$. bronchiseptica (white bars) opsonized with either two different concentrations $(2.5 \%$ and $5 \%)$ of post-immune serum or $5 \%$ of pre-immune serum from a pertussis vaccinated donor. Data represent the mean \pm S.D. of four experiments with PMN from different donors. AU: arbitrary units. 


\section{Discussion}

Many reports have documented the increase of $B$. pertussis incidence even in highly vaccinated populations [9], [11] and [13]. The causes for this increase are still under investigation. However, it has been proposed that, in addition to the waning immunity in adolescents and adults [33] and [34], who could then be reinfected and serve as source of pathogen to young infants, $B$. pertussis may have evolved in a way that is less sensitive to vaccine-induced immunity [35] and [36]. An increasing body of evidence collected in different countries supports this hypothesis [14], [15], [16], [17], [18] and [19]. Here we report the first study on B. pertussis molecular epidemiology in Buenos Aires, showing a clear divergence between the strain used in vaccine production and clinical isolates.

Samples from pediatric patients with pertussis symptomathology were collected during 6 years. Twenty-five percent of the total number of samples proved to be Bordetella by PCR ( $98 \%$ positive for $B$. pertussis and $2 \%$ positive for $B$.

bronchiseptica) but only $7.5 \%$ (30 isolates) were culture positive. The difference between the initial detection of Bordetella positives by PCR and the number of strains that could actually be cultured may be due not only to the difference in the sensitivity of the techniques used but also to the decrease in bacterial viability that may occur after sampling and during transport.

From the 30 Bordetella isolates, 28 were identified as $B$. pertussis and two as $B$. bronchiseptica. No differences in the virulent state of the isolates could be detected. However, an interesting difference was observed in the bacterial LPS pattern in the $B$. pertussis isolates. A shift from the classical pattern of two bands (LPS A and LPS B) to only one band (LPS A) was observed (Fig. 1) in some of the isolates. This enrichment in LPS A with respect to LPS $B$ is consistent with the hypothesis that the membrane-distal region of the LPS molecule (i.e. the LPS A), which is in intimate contact with the bacterial environment and might have an important role in host-bacterial interactions, would be expressed at a higher level within the host [37]. Harvill et al. [30] demonstrated that $\Delta w / b$ mutants of $B$. pertussis which do not produce the LPS A form, were defective in colonization of the respiratory tracts of BALB/c mice. Furthermore, it was reported that $B$. pertussis LPS mutants lacking even one of the sugars in the terminal trisaccharide were bound and aggregated by surfactant protein A (SP-A). Phagocytosis by human monocytes was enhanced on LPS mutants that were able to bind SP-A, but not on wild-type bacteria [38]. The authors concluded that the LPS of wildtype $B$. pertussis shields the bacteria from SP-A-mediated clearance, possibly by sterically limiting access to the lipid $A$ region. Taken together, these results seem to indicate that LPS A is an essential form of LPS for $B$. pertussis infection. If we assume that a selection pressure towards more infective genotypes operates within the bacterial population then the enrichment of LPS A-expressing bacteria can be expected. Thus, it would be of interest to study whether vaccination has any role in this selection.

We also compared isolates and the vaccine strain following the recommended approach of sequencing the genes that code for two proteins that confer protective immunity in animals and humans, pertactin (Prn) and pertussis toxin (Ptx). In addition, we used a more discriminative technique, the PCR fingerprinting, to establish the relatedness among the isolates and in relation to the vaccine strain.

Several reports from Europe and USA [9] and [10] documented in that countries the prevalence of isolates expressing Prn and Ptx variants not present in vaccine. Four PtxS1 types (PtxS1A-D) have been described, from which PtxS1A was most 
frequently found as a prevalent variant. By contrast, the acellular and cellular vaccines currently in use contain PtxS1B and PtxS1D but not PtxS1A [39]. Regarding pertactin, many variants have been described, of which Prn2 was mostly observed (vaccines contain Prn1). The alleles prn1 and ptxS1B or D have been termed by Cassiday et al. [40] old alleles and the strains possessing both alleles, old strains. Strains possessing prn2 or 3 and ptxS1A alleles have been assigned to the group of new strains, and those that possess one new and one old prn or ptxS1 to the group of transitional strains. In The Netherlands, Finland, Italy, France, and United States it was observed that the strains possessing both an old prn and an old ptxS1 allele have origins prior to the 1970 s. In contrast new strains having prn2, prn3 and ptxS1A were first detected from the mid1980 s till to now in all the countries mentioned above [10], [17] and [41].

In Buenos Aires the majority of circulating bacteria possesses $p t x S 1 A$ and $p r n 2$ allelic variants. These allelic frequencies found in Argentina almost exactly match those found in distant countries at the Northern hemisphere, arguing in favor of vaccine-driven selection pressure. Since vaccine was introduced in Argentina in the 1950s, our observations probably reflect the equilibrium allelic frequencies reached at present, after more than 40 years of selection; thereby we did not observe changes between 1997 and 2003.

The divergences between the vaccine strain (PtxS1D and Prn1) and current isolates with the prevalence of the PtxS1A and Prn2 variants seem to be critical in protection against pertussis [14], [15] and [42]. The results obtained in animal models first by King et al. [15] and recently confirmed independently by Gzyl et al. [14], showed that whole cell vaccines were less effective against strains that carried non-vaccine-type Prn variants compared to strains that carried the vaccine-type. These results, together with the finding that antibodies against both Ptx and Prn are critical in protection against pertussis [36] and [42], also seem to support the hypothesis of vaccine-driven bacterial selection.

In agreement with a previous report, we observed that PCR-fingerprinting employing enterobacterial repetitive consensus primers (ERIC-PCR) was poorly discriminatory. However, when BOXAR1 primers were used the techniques proved to be appropriate since it has a good discriminatory power and reproducibility. In addition to the PtxS1 and Prn differences referred to above, BOXAR1-PCR underscored the genotypic differences between the vaccine and circulating strains. Fingerprints of genomic DNA from all $B$. pertussis isolates yielded a major cluster containing 13 clinical isolates and a few small ones, all with DNA fragment sizes ranging from 0.2 to $4.0 \mathrm{~kb}$ (Fig. 1). Although there were small differences among the profiles of $B$. pertussis isolates, the vaccine strain profile was significantly distinct from all of them.

We found $B$. bronchiseptica in two of the patients (19 months and 12 years old) that were investigated for Bordetellae infection, none of them known to be in contact with infected animals. This microorganism has been rarely found in humans and only in adult patients with known exposure to infected animals [43]. We wondered whether lack of cross protection by the vaccine was the cause for these atypical infections. Our results show that whole cell pertussis vaccineinduced antibodies that reacted with $B$. bronchiseptica components, and that these antibodies proved capable of inducing opsonophagocytosis of $B$. bronchiseptica by professional phagocytes, a potent immune mechanism against these bacteria [26] and [27]. This result is in agreement with those published by Kirimanjeswara et al. [44] who observed that $B$. bronchiseptica but not $B$. pertussis is rapidly cleared by adoptively transferred serum antibodies. Kirimanjeswara et al. [45] showed that $B$. pertussis blocked migration of 
neutrophils and inhibit their recruitment to the lung during the first week of infection by a pertussis toxin-dependent mechanism. Consequently PTx allows $B$. pertussis to avoid rapid antibody-mediated clearance, a mechanism not shared by $B$. bronchiseptica since it does not express pertussis toxin.

Although more research will be necessary to rule out the possibility of inefficient cross protection between these two Bordetella species, other phenomena might explain these infections with $B$. bronchiseptica, for instance, waning immunity for the 12-year-old patient. However, we cannot provide a satisfactory explanation for the 19-month-old patient who had the vaccination schedule completed according to his age.

In summary, the results of this study show that $B$. pertussis circulating bacteria were different from the strain used for vaccine production and similar to those previously found responsible for most pertussis outbreaks in other parts of the world. This result and the fact that $B$. bronchiseptica was found infecting the pediatric population should be considered for the development of an improved vaccine.

\section{Acknowledgements}

This work was supported by grant Antorchas Foundation and IFS-B/2672-2 (Sweden) to D.H., CONICET (Argentina) and CICBA (Argentina). D.B., J.F. and F.S. were supported by CONICET. D.H. is a member of the Scientific Career of CICBA. M.E.R. is member of the Scientific Career of CONICET. 


\section{References}

[1] G. Gerlach, F. von Wintzingerode, B. Middendorf and R. Gross, Evolutionary trends in the genus Bordetella, Microbes Infect 3 (2001), pp. 61-72.

[2] J.M. Musser, E.L. Hewlett, M.S. Peppler and R.K. Selander, Genetic diversity and relationships in populations of Bordetella spp., J Bacteriol 66 (1986), pp. 230-237.

[3] J.G. Coote, Environmental sensing mechanisms in Bordetella, Adv Microb Physiol 44 (2001), pp. $141-181$.

[4] A. Van der Zee, H. Groenendijk, M. Peeters and F.R. Mooi, The differenciation of B. parapertussis and $\mathrm{B}$. bronchiseptica from man and animals as determined by DNA polymorphism mediated by two different insertion sequence elements suggests their phylogenetic relationship, Int J Syst Bacteriol 46 (1996), pp. 640-647.

[5] D.A. Bemis, W.R. Shek and C.B. Clifford, Bordetella bronchiseptica infection of rats and mice, Comp Med 53 (2003), pp. 11-20.

[6] P. Gueirard, C. Weber, A. LeCoustumier and N. Guiso, Human Bordetella bronchiseptica infection related to contact with infected animals, persistence of the bacteria in the host, J Clin Microbiol 33 (1995), pp. 2002-2006.

[7] V. Lo Re III, P.J. Brennan, J. Wadlin, R. Weaver and I. Nachamkin, Infected branchial cleft cyst due to Bordetella bronchiseptica in an immunocompetent patient, J Clin Microbiol 39 (2001), pp. 4210-4212.

[8]B.F. Woolfrey and J.A. Moody, Humans infections associated with Bordetella bronchiseptica, ] Clin Microbiol 4 (1991), pp. 243-255.

[9] H.E. de Melker, M.A. Conyn-van Spaenddonck, H.C. Rumke, J.K. van Wijngaarden, F.R. Mooi and J.F.P. Schellekens, Pertussis in The Netherlands: an outbreak despite high levels of immunization with whole cell vaccine, Emerg Infect Dis 3 (1997), pp. 175-178.

[10] T.H. Hardwick, P. Cassiday, R.S. Weyant, K.M. Bisgard and G.N. Sanden, Changes in predominance and diversity of genomic subtypes of Bordetella pertussis isolated in the United States, 1935 to 1999, Emerg Infect Dis 8 (2002), pp. 44-49.

[11] G. De Serres, N. Bouliane, M. Douville-Fradet and B. Duval, Pertussis in Quebec: ongoing epidemic since the late, Can Commun Dis Rep 15 (1980), pp. 45-48.

[12] P. Olin, L. Gustafsson, L. Barreto, L. Hessel, T.C. Mast and A.V. Rie et al., Declining pertussis incidence in Sweeden following the introduction of acellular pertussis vaccine, Vaccine 21 (2003), pp. 2024-2030.

[13] R. Andrews, A. Herceg and C. Roberts, Pertussis notifications in Australia, 1991 to 1997, Commun Dis Intell 21 (1997), pp. 145-148.

[14] A. Gzyl, E. Augustynowicz, G. Gniadek, D. Rabczenko, G. Dulny and J. Lusarczyk, Sequence variation in pertussis S1 subunit toxin and pertussis genes in Bordetella pertussis strains used for the whole-cell pertussis vaccine produced in Poland since 1960: Efficiency of the DTwP vaccine-induced immunity against currently circulating B. pertussis isolates, Vaccine 22 (2004), pp. 2122-2128.

[15] A.J. King, G. Berbers, H.F. van Oirschot, P. Hoogerhout, K. Knipping and F.R. Mooi, Role of the polymorphic region 1 of the Bordetella pertussis protein pertactin in immunity, Microbiology 147 (2001), pp. 2885-2895.

[16] F.R. Mooi, H. van Oirschot, K. Heulvelman, H.G.J. van der Heide, W. Gaastra and R.J.L. Willems, Polymorphism in Bordetella pertussis virulence factors P.69/pertactin and pertussis toxin in The Netherlands: temporal trends and evidence for vaccine-driven evolution, Infect Immun 66 (1998), pp. 670-675. 
[17] F.R. Mooi, H. Qiushui, H. van Oirschot and J. Mertsola, Variation in the Bordetella pertussis virulence factors pertussis toxin and pertactin in vaccine strains and clinical isolates in Finland, Infect Immun 67 (1999), pp. 3133-3134.

[18] F.R. Mooi, H. Hallander, C.H. Wirsing von Köning, B. Hoet and N. Guiso, Epidemiological typing of Bordetella pertussis isolates: recommendations for a standard methodology, Eur Clin Infect Dis J 19 (2000), pp. 174-181.

[19] J. Fiett, I. Letowska, M. Gniadkowski and W. Hryniewicz, The new strategy for allele identification of the genes coding for pertussis subunit S1 (ptx S1) and pertactin (prn) in Bordetella pertussis, J Microbiol Methods 55 (2003), pp. 651-666.

[20] D. Hozbor, F. Fouque and N. Guiso, Detection of Bordetella bronchiseptica by the polymerase chain reaction, Res Microbiol 150 (1999), pp. 333-341.

[21] E. Grimprel, P. Begue, I. Anjak, F. Betsou and N. Guiso, Comparison of polymerase chain reaction, culture and western immunoblot serology for diagnosis of Bordetella pertussis infection, J Clin Microbiol 31 (1993), pp. 2745-2750.

[22] J. Versalovic, M. Schneider, F.J. De Bruijn and J. Lupski, Genomic fingerprinting of bacteria using repetitive sequence-based polymerase chain reaction, Methods Mol Cell Biol 5 (1994), pp. 25-40.

[23] P. Gueirard and N. Guiso, Virulence of Bordetella bronchiseptica: role of adenylate cyclasehemolysin, Infect Immun 61 (1993), pp. 4072-4078.

[24] C. Valverde, D.F. Hozbor and A. Lagares, Rapid preparation of affinity-purified lipopolysaccharide samples for electrophoretic analysis, Biotechniques 22 (1997), pp. 230-235.

[25] R. Repp, T. Valerius, A. Sendler, M. Gramatzki, H. Iro and J.R. Kalden et al., Neutrophils express the high affinity receptor for IgG (Fc gamma RI, CD64) after in vivo application of recombinant human granulocyte colony-stimulating factor, Blood 78 (1991), pp. 885-889.

[26] M.E. Rodriguez, S.M.M. Hellwig, D. Hozbor, W.L. van der Pol and J.G.J. van de Winkel, Fc receptor-mediated immunity against Bordetella pertussis, J Immunol 167 (2001), pp. 6545-6551.

[27] M.E. Rodriguez, W.L. van der Pol and J.G.J. van de Winkel, Flow-cytometry-based phagocytosis assay for sensitive detection of opsonic activity of pneumococcal capsular polysaccharide antibodies in human sera Fc receptor-mediated immunity against Bordetella pertussis, J Immunol Methods 252 (2001), pp. 33-34.

[28] B. Arico and R. Rappuoli, Bordetella parapertussis and Bordetella bronchiseptica contain transcriptionally silent pertussis toxin genes, J Bacteriol 169 (1981), pp. 2847-2853.

[29] P. Gueirard, K. Le Blay, A. Le Coustumier, R. Chaby and N. Guiso, Variation in Bordetella bronchiseptica lipopolysaccharide during human infection, FEMS Microbiol Lett 15 (1998), pp. 331337.

[30] E.T. Harvill, A. Preston, P.A. Cotter, A. Allen, D.J. Maskell and J.F. Miller, Multiples roles for Bordetella lipopolysaccharide molecules during respiratory tract infection, Infect Immun 68 (2000), pp. 6720-6728.

[31] F. Sisti, J. Fernández, M.E. Rodríguez, A. Lagares, N. Guiso and D.F. Hozbor, In vitro and in vivo characterization of a Bordetella bronchiseptica mutant strain with deep rough lipopolysaccharide structure, Infect Immun 70 (2002), pp. 1791-1798.

[32] J. van Putten, Phase variation of lipopolysaccharide directs interconversion of invasive and immuno-resistant phenotypes of Neisseria gonorrhoeae, EMBO J 12 (1993), pp. 4043-4051.

[33] F. Romano, M.L. Quintana, C. de Dahaer, L. Bogni, P. Thumas and M. Moreschi et al., Brote de coqueluche en Esquel, Arch Argent Pediatr 100 (2002), pp. 11-18.

[34] J. Hoey, Pertussis in adults, CMAJ 168 (2003), pp. 453-454. 
[35] F.R. Mooi, I.H. van Loo and A.J. King, Adaptation of Bordetella pertussis to vaccination: a cause for its reemergence?, Emerg Infect Dis 7 (2001), pp. 526-528.

[36] Q. He, J. Makinen, G. Berbers, F.R. Mooi, M.K. Viljanen and H. Arvilommi et al., Bordetella pertussis protein pertactin induces type-specific antibodies: one possible explanation for the emergence of antigenic variants?, J Infect Dis 187 (2003), pp. 1200-1205.

[37] A. Preston and D.J. Maskell, Molecular genetics and role in infection of environmentally regulated lipopolysaccharide expression, Int J Med Microbiol 292 (2002), pp. 7-15.

[38] L.M. Schaeffer, F.X. McCormack, H. Wu and A.A. Weiss, Bordetella pertussis lipopolysaccharide resists the bactericidal effects of pulmonary surfactant protein, J Immunol 173 (2004), pp. 19591965.

[39] S.C.M. van Amersfoorth, L.M. Schouls, H.G.J. van der Heide, A. Advani, H.O. Hallander and K. Bondeson et al., Analysis of Bordetella pertussis population in European countries with different vaccination policies, J Clin Microbiol 43 (2005), pp. 2837-2843.

[40] P. Cassiday, G. Sanden, K. Heuvelman, F. Mooi, M. Bisgard and T. Popovic, Polymorphism in Bordetella pertussis pertactin and pertussis toxin virulence factors in the United States, 1935-1999, J Infect Dis 182 (2000), pp. 1402-1408.

[41] P. Stefanelli, L. De Marzi, L. Robino, D. Roman, F. Basso and P. D'Orazio et al., Characterization of Bordetella pertussis strains of recent isolation, Microbiologica 22 (1999), pp. 187-194.

[42] K. Kamachi, T. Konda and Y. Arakawa, DNA vaccine encoding pertussis toxin S1 subunit induces protection against Bordetella pertussis in mice, Vaccine 21 (2003), pp. 4609-4615.

[43] B.F. Woolfrey and J.A. Moody, Humans infections associated with Bordetella bronchiseptica, J Clin Microbiol 4 (1991), pp. 243-255.

[44] G.S. Kirimanjeswara, P.B. Mann and E.T. Harvill, Role of antibodies in immunity to Bordetella infections, Infect Immun 71 (2003), pp. 1719-1724.

[45] G.S. Kirimanjeswara, L. Agosto, M. Kennett, O. Bjornstad and E.T. Harvill, Pertussis toxin inhibits neutrophils recruitment to delay antibody-mediated clearance of Bordetella pertussis, J Clin Invest 10 (2005), pp. 1172-1180. 